\title{
A combination of inhibitors of glycolysis, glutaminolysis and de novo fatty acid synthesis decrease the expression of chemokines in human colon cancer cells
}

\author{
ALEJANDRO SCHCOLNIK-CABRERA ${ }^{1}$, GUADALUPE DOMINGUEZ-GÓMEZ ${ }^{1}$, \\ ALMA CHÁVEZ-BLANCO ${ }^{1}$, MARISOL RAMÍREZ-YAUTENTZI ${ }^{1}$, ROCÍO MORALES-BÁRCENAS ${ }^{1}$, \\ JOSÉ DÍAZ-CHÁVEZ ${ }^{1}$, LUCÍA TAJA-CHAYEB ${ }^{1}$ and ALFONSO DUEÑAS-GONZÁLEZ ${ }^{1,2}$ \\ ${ }^{1}$ Basic Research Division, National Cancer Institute; ${ }^{2}$ Biomedical Research Unit in Cancer, Biomedical \\ Research Institute, National Autonomous University of Mexico, Mexico City 14080, Mexico
}

Received April 19, 2019; Accepted September 24, 2019

DOI: $10.3892 / \mathrm{ol} .2019 .11008$

\begin{abstract}
Lonidamine, 6-Diazo-5-oxo-L-norleucine (DON) and orlistat are well known inhibitors of glycolysis, glutaminolysis and of de novo fatty acid synthesis, respectively. Although their antitumor effects have been explored in detail, the potential inhibition of the malignant metabolic phenotype and its influence on the expression of chemokines and growth factors involved in colon cancer, have not been previously reported to the best of our knowledge. In the present study, dose-response curves with orlistat, lonidamine or DON were generated from cell viability assays conducted in SW480 colon cancer cells. In addition, the synergistic effect of these compounds was evaluated in SW480 human colon cancer cells. The determination of the doses used for maximum synergistic efficacy led to the exploration of the mRNA levels of the target genes hexokinase-2 (HK2), glutaminase-1 (GLS-1) and fatty acid synthase $(F A S N)$ in human SW480 and murine CT26.WT colon cancer cells. The cell viability was evaluated following transfection with small interfering (si)RNA targeting these genes and was assessed with trypan blue. The expression levels of chemokines and growth factors were quantified in the supernatant of SW480 cells with LEGENDplex ${ }^{\mathrm{TM}}$. The combination of lonidamine, DON and orlistat resulted in a synergistic cytotoxic effect and induced the transcription of the corresponding gene targets but their corresponding proteins were actually downregulated. The downregulation of the expression levels of $H K 2, G L S-1$ and $F A S N$ following transfection of the cells with the corresponding siRNA sequences decreased their viability. The treatment significantly reduced the expression levels of
\end{abstract}

Correspondence to: Dr Alfonso Dueñas-González, Basic Research Division, National Cancer Institute, 22 San Fernando Avenue, Tlalpan, Mexico City 14080, Mexico

E-mail: alfonso_duenasg@yahoo.com

Key words: cancer metabolism, chemokines, small interfering RNA blockade, lonidamine, 6-Diazo-5-oxo-L-norleucine, orlistat
9 chemokines [interleukin-9, C-X-C motif chemokine ligand (CXCL) 10, eotaxin, chemokine ligand (CCL) 9, CXCL5, CCL20, CXCL1, CXCL11 and CCCL4] and one growth factor (stem cell factor). These changes were associated with decreased phosphorylated-nuclear factor $\mathrm{\kappa B}-\mathrm{p} 65$. The data demonstrate that lonidamine, DON and orlistat in combination reduce the expression levels of chemokines and growth factors in colon cancer cells. Additional research is required to investigate the exact way by which both tumor and stromal cells regulate the expression levels of chemokines and growth factors.

\section{Introduction}

Cancer cells reprogram their metabolism to increase energy and nutrient depots for their continuous biosynthetic requirements (1). While healthy cells follow rigorous metabolic pathways that secure the balance between anabolism and catabolism, cancer cells override these molecular checkpoints and increase their anabolism, at the cost of the catabolism of the host (2).

The malignant metabolic reprogramming occurs at the three main pathways: Glycolysis, glutaminolysis and de novo fatty acid (FA) synthesis. Therefore, cancer cells commonly overexpress hexokinase-II (HK2), glutaminase-1 (GLS1) and fatty acid synthase (FASN) (3). In addition to their energetic requirements, malignant cells require macromolecule synthesis, including synthesis of nucleotides, amino acids and lipids. Glucose and glutamine supply the majority of the necessary carbon and nitrogen atoms required for the synthesis of macromolecules. These atoms further serve as reducing equivalents in order to support cell growth $(4,5)$. Regarding lipogenesis, malignant cells synthesize de novo FA instead of removing them from circulation. Therefore, cancer cells frequently overexpress FASN (6). Regarding the de novo synthesis of FA, both glucose and glutamine supply citrate. Glucose is converted to acetyl-CoA in the mitochondria in order to produce citrate in the tricarboxylic acid cycle, whereas glutamine maintains citrate production by supplying the carbon in the form of mitochondrial oxaloacetate. 
Therefore, the metabolism of glutamine and glucose supports the production of acetyl-CoA and NADPH required for FA synthesis (5).

A previous report using this combination of orlistat+loni damine+6-Diazo-5-oxo-L-norleucine (OLD) in vitro showed that while it inhibits the growth of number of malignant cell lines, the effect is minor in primary lung fibroblasts suggesting that OLD preferentially targets malignant cells (7). Moreover, in syngeneic and allogenic mouse models, this treatment exerts an antitumor effect without affecting the weight of treated mice. Nevertheless, serum chemokines were not measured (8). In the present study, orlistat, lonidamine and DON were employed, which inhibited FASN, HK2 and GLS1, respectively, and analyzed the transcriptional and protein levels of their corresponding drug targets by reverse transcription PCR. Using small interfering (si)RNA interference of the same targets, cellular viability was measured in the absence or presence of specific compounds inhibiting the remaining energetic pathways. In addition, the study investigated whether the metabolic inhibition may influence the expression of chemokines and growth factors associated with colon cancer.

\section{Materials and methods}

Cell lines. The human colon adenocarcinoma SW480 and murine CT26.WT cell lines were obtained from the American Type Culture Collection. Both cell lines were cultured at $37^{\circ} \mathrm{C}$ in a humidified atmosphere containing $5 \% \mathrm{CO}_{2}$, in DMEM/F12 (SW480) or RPMI-1640 (CT26.WT) in the presence of $10 \%$ fetal bovine serum and $1 \%$ antibiotic/antimycotic solution (all from Invitrogen; Thermo Fisher Scientific, Inc.).

Drug treatments. Orlistat (Psicofarma, S.A., De C.V.), lonidamine and DON were dissolved in absolute ethanol, dimethyl sulfoxide, and culture medium without serum, respectively. All drugs with the exception of orlistat and all vehicles were from Sigma-Aldrich; Merck KGaA. Drug treatments were freshly prepared for each experiment.

SW480 cell viability assays and identification of inhibitory concentrations (ICs). A total of $5 \times 10^{4}$ cells/well were seeded in 6-well plates with $2 \mathrm{ml}$ complete medium and were allowed to attach to the bottom of the surface overnight. Subsequently, complete medium containing either orlistat, lonidamine or DON was added at increasing doses every $24 \mathrm{~h}$ until $72 \mathrm{~h}$. Control cells were treated with the vehicle used for each drug, using the same volume as of that of the highest evaluated dose. The cells were washed once with 1X PBS and detached with a $0.5 \%$ trypsin/2\% EDTA solution. Cell viability was evaluated by the trypan blue exclusion assay. Briefly, the cells were gently mixed at $1: 1$ ratio with trypan Blue Stain $(0.4 \%$; Gibco; Thermo Fisher Scientific, Inc.) for $5 \mathrm{sec}$ and placed in a TC10 $10^{\mathrm{TM}}$ Automated Cell Counter (Bio-Rad Laboratories, Inc.) at room temperature. The cytotoxic effect was expressed as the percentage of cell viability relative to the control cells. The resulting data were analyzed in the SigmaPlot software (version 10.0; Systat Software, Inc.). The percentage of growth inhibition was estimated and the $\mathrm{IC}_{20}-\mathrm{IC}_{50}$ values were obtained from the survival curves.
Drug treatment. Increasing doses of orlistat $\left(\mathrm{IC}_{20}, \mathrm{IC}_{30}, \mathrm{IC}_{40}\right.$ and $\mathrm{IC}_{50}$ ) were combined with their respective increasing doses of lonidamine $\left(\mathrm{IC}_{20}, \mathrm{IC}_{30}, \mathrm{IC}_{40}\right.$ and $\left.\mathrm{IC}_{50}\right)$ and $\mathrm{DON}\left(\mathrm{IC}_{20}, \mathrm{IC}_{30}\right.$, $\mathrm{IC}_{40}$ and $\mathrm{IC}_{50}$ ). The resulting mixed solutions were incubated for $72 \mathrm{~h}$ with $5 \times 10^{4} \mathrm{SW} 480$ cells/well, as previously stated. The pharmacological interaction was determined with the combination index (CI) method from the formula of Chou and Talalay using the Calcusyn software (version 2.0; Biosoft) (9). The CI values used to assign the pharmacological interaction are shown in Table I. The synergistic combination at the lowest doses was selected for further experiments.

Reverse transcription-quantitative $(R T-q) P C R$ of the target enzymes. A total of $5 \times 10^{4}$ (SW480) or $3 \times 10^{4}$ (CT26.WT) cells were seeded and treated as stated above. Following $72 \mathrm{~h}$ of treatment, the cells were detached and total RNA isolation was carried out using TRIzol (Thermo Fisher Scientific, Inc.). A total of $1 \mu \mathrm{g}$ of total RNA was used for cDNA synthesis at $45^{\circ} \mathrm{C}$ with the GeneAmp RNA PCR Core kit (Applied Biosystems; Thermo Fisher Scientific, Inc.). cDNA was mixed with the iQ SYBR Green SuperMix (Bio-Rad Laboratories, Inc.) according to the manufacturer's protocol. qPCR reactions were run using an ABI Prism 7000 (Applied Biosystems; Thermo Fisher Scientific, Inc.). The qPCR cycling conditions were as follows: Initial denaturation for $10 \mathrm{~min}$ at $95^{\circ} \mathrm{C}$ followed by 40 cycles of $30 \mathrm{sec}$ at $95^{\circ} \mathrm{C}, 30 \mathrm{sec}$ at $60^{\circ} \mathrm{C}$ and $30 \mathrm{sec}$ at $72^{\circ} \mathrm{C}$. The data were analyzed using the $2^{-\Delta \Delta \mathrm{Cq}}$ method (10) and reported as the fold-change in gene expression normalized to the expression of the endogenous control gene hypoxanthine phosphoribosyltransferase 1 (HPRT1) (for SW480), or glyceraldehyde-3-phosphate dehydrogenase $(G A P D H)$ (for CT26.WT). The primers used for detection of the human genes were as follows: HPRT1 forward, 5'-GAA CCTCTCGGCTTTCCCG-3' and reverse, 3'-CACTAATCA CGACGCCAGGG-5'; HK2 forward, 5'-GCAGAAGGTTGA CCAGTATC-3' and reverse, 5'-TGGAGTGGACCTCAC AAA-3'; GLS1 forward, 5'-CTCTTCCGAAAGTGTGTG AG-3' and reverse, 5'-CAGGTCTGGGTTTGACTTG-3'; FASN forward, 5'-CGCTCGGCATGGCTATCTC-3' and reverse, 5'-CTCGTTGAACGCATCCA-3'. The detection of the mouse genes was performed with the following primers: $H K 2$ forward, 5'-GCAGAAGGTTGACCAGTATC-3' and reverse, 5'-CGGAGTTGACCTCACAAAG-3'; GLS1 forward, 5'-ACAGGAGCGTATCCCTATC-3' and reverse, 5'GTTGCT GCTCACACACTT-3'; FASN forward, 5'-GGCACTGACTGT CTGTTTTCCA-3' and reverse, 5'-TTAATTGTGGGATCA GGAGAGCAT-3' and GAPDH forward 5'-GTGGAGTCA TACTGGAACATGTAG-3' and reverse 5'-AATGGTGAA GGTCGGTGTG-3'. The latter gene was used for normalization purposes. The annealing temperature was $60^{\circ} \mathrm{C}$ for all reactions.

siRNA inhibition of target enzymes. A total of $8 \times 10^{4}$ (SW480) and $4 \times 10^{4}$ (CT26.WT) cells were seeded in 6-well plates to achieve $50 \%$ confluent cultures at the time of transfection. A total of 12.5 pmol siRNA was used for knockdown of the $H K 2$, FASN and GLS1 genes (HK2 cat. no. 4390824 ID s6562; FASN cat. no. 4390824 ID 55031; and GLS1 cat. no. 4392420 ID s5838) in SW480 cells. In CT26.WT cells, 12.5 pmol (FASN cat. no. 4390771 ID 565865), GLS1 (cat. no. AM16708 ID 
Table I. CI values, recommended symbols and descriptions for determining synergism, antagonism or addition using the Chou-Talalay formula.

\begin{tabular}{lcl}
\hline Range of CI & Symbol & \multicolumn{1}{c}{ Description } \\
\hline$<0.1$ & +++++ & Very strong synergism \\
$0.1-0.3$ & ++++ & Strong synergism \\
$0.3-0.7$ & +++ & Synergism \\
$0.7-0.85$ & ++ & Moderate synergism \\
$0.85-0.9$ & + & Slight synergism \\
$0.9-1.1$ & \pm & Nearly additive \\
$1.1-1.2$ & - & Slight antagonism \\
$1.2-1.45$ & -- & Moderate antagonism \\
$1.45-3.3$ & --- & Antagonism \\
$3.3-10$ & ---- & Strong antagonism \\
$>10$ & ----- & Very strong antagonism \\
\hline
\end{tabular}

CI, combinatory index.

501056) and 25 pmol (HK2 cat. no. AM16708 ID 159330) siRNA (all from Ambion; Thermo Fisher Scientific, Inc.) sequences were diluted in $100 \mu 1$ Opti-MEM-I reduced serum medium (Gibco; Thermo Fisher Scientific, Inc.). Lipofectamine ${ }^{\circledR}$ RNAiMAX transfection reagent ( $4 \mu \mathrm{l}$; Invitrogen; Thermo Fisher Scientific, Inc.) was diluted in $100 \mu$ l Opti-MEM-I. The mixtures were pooled and incubated for $20 \mathrm{~min}$ at room temperature. Silencer select negative control No. 1 siRNA (cat. no. 4390844; Ambion; Thermo Fisher Scientific, Inc.) was used as a negative control for both cell lines. The mixture was added to each well in $1 \mathrm{ml}$ Opti-MEM-I and replaced following $6 \mathrm{~h}$ of incubation with complete fresh medium or complete medium in the presence of drugs. This protocol was used for the experiments combining siRNA and drug treatment. For both cell lines, one siRNA sequence was added per time and the effects were compared with the control without siRNA, the silencer negative control, the siRNA inhibition groups and the drug groups in the presence of siRNA treatment. Fresh complete medium was provided every $24 \mathrm{~h}$ for a total period of $72 \mathrm{~h}$. The compounds were added at their $\mathrm{IC}_{40}$ doses when the medium was changed. Following $72 \mathrm{~h}$, the compounds were removed and viability was evaluated with trypan blue as described above. The cytotoxic effect was expressed as a percentage of cell viability relative to the untransfected control cells.

Total protein extraction, western blot analysis and densitometry. Following either OLD or siRNA treatments, total protein was extracted using radio-immunoprecipitation buffer $(150 \mathrm{mM} \mathrm{NaCl} ; 1.0 \%$ IGEPAL CA630; $0.5 \%$ sodium deoxycholate; $0.1 \%$ SDS and $50 \mathrm{mM}$ Tris, $\mathrm{pH}$ 8.0) in the presence of proteinase inhibitors (cat. no. p8340; Sigma-Aldrich; Merck KGaA). The protein concentration was determined using the bicinchoninic acid assay. A total of $30 \mu \mathrm{g}$ of protein was separated by $10 \%$ SDS-PAGE and transferred onto a PVDF membrane (cat. no. 162-0177; Bio-Rad Laboratories, Inc.). The membrane was blocked with 5\% skimmed milk in 1X PBS ( $1 \mathrm{~h}$ at room temperature) and subsequently incubated with antibodies against GLS1 (cat. no. sc-100533; 1:500; Santa Cruz Biotechnology, Inc.); HK2 (cat. no. sc-6521; 1:200; Santa Cruz Biotechnology, Inc.); FASN (cat. no. sc-55580; 1:500; Santa Cruz Biotechnology, Inc.), the phosphorylated p65 subunit of nuclear factor (NF)кB (cat. no. 6956; 1:10,000; Cell Signaling Technology, Inc.), and anti-actin peroxidase (cat. no. A3854; 1:10,000; Sigma-Aldrich; Merck KGaA) in blocking solution (5\% skimmed milk in TBS $/ 0.1 \%$ Tween 20 ), overnight at $4^{\circ} \mathrm{C}$. The secondary antibodies used were the following: Donkey anti-goat for HK2 (cat. no. sc-2020); and bovine anti-mouse for GLS1, FASN, and the phosphorylated p65 subunit of NF-kB (cat. no. sc-2371). The secondary antibodies were diluted at 1:1,000 ( $1 \mathrm{~h}$ at room temperature). The protein bands were visualized using the Clarity Western Enhanced Chemiluminescence Substrate (cat. no. 1705060; Bio-Rad Laboratories, Inc.). The bands were quantified by densitometry using the Image $\mathbf{J}$ software, version 1.50 (National Institute of Health).

Flow cytometry evaluation of chemokines and growth factors in the supernatant. A total of $5 \times 10^{4}$ SW480 cells were seeded in 6-well plates and treated as stated above. The supernatant was recovered every $24 \mathrm{~h}$ until the end of the treatment and subsequently centrifuged $\left(650 \mathrm{x} \mathrm{g}, 20 \mathrm{~min}, 4^{\circ} \mathrm{C}\right)$ and stored at $-20^{\circ} \mathrm{C}$ until further use. The LEGEND plex ${ }^{\mathrm{TM}}$ Human Proinflammatory Chemokine Panel (cat. no. 740003) and Human Growth Factor Panel (cat. no. 740180) were employed to quantify the expression levels of chemokines and growth factors in the supernatant, following the manufacturer's protocol. The analysis was performed with the BD FACSCanto $^{\mathrm{TM}}$ II flow cytometer with BD FACSDiva ${ }^{\mathrm{TM}}$ software (BD Biosciences).

Statistical analysis. A total of 3 independent experiments were performed in triplicate per assay and the data were expressed as the mean \pm standard deviation. The data were analyzed with GraphPad Prism V6 software (GraphPad Software Inc.). Significant differences were analyzed with the Student's t test for data derived from RT-qPCR, densitometry and growth factor/chemokine expression analysis. Regarding dose-response curves and siRNA inhibition experiments, the significance of the differences was determined with one-way analysis of variance and Tukey post hoc correction. $\mathrm{P}<0.05$ was considered to indicate a statistically significant difference.

\section{Results}

Viability inhibition. Treatment of human and murine cells with orlistat, DON and lonidamine exerted a dose-dependent effect on cell viability reduction at the concentrations tested. These differences were statistically significant compared with those noted in vehicle-treated control cells $(\mathrm{P}<0.05$; Fig. 1A-C $)$.

Determination of the $I C_{20}-I C_{50}$. Following treatment of SW480 cells with the drugs, the $\mathrm{IC}_{20}-\mathrm{IC}_{50}$ doses were obtained using SigmaPlot software. These values are shown in Table II. Subsequently, the cells were treated with the different ICs of the three drugs in combination. A significant difference was noted with regard to inhibition of cell proliferation for each IC $(\mathrm{P}<0.05)$. It is interesting to note that the cell viability dropped below $20 \%$ when the $\mathrm{IC}_{50}$ was used. 

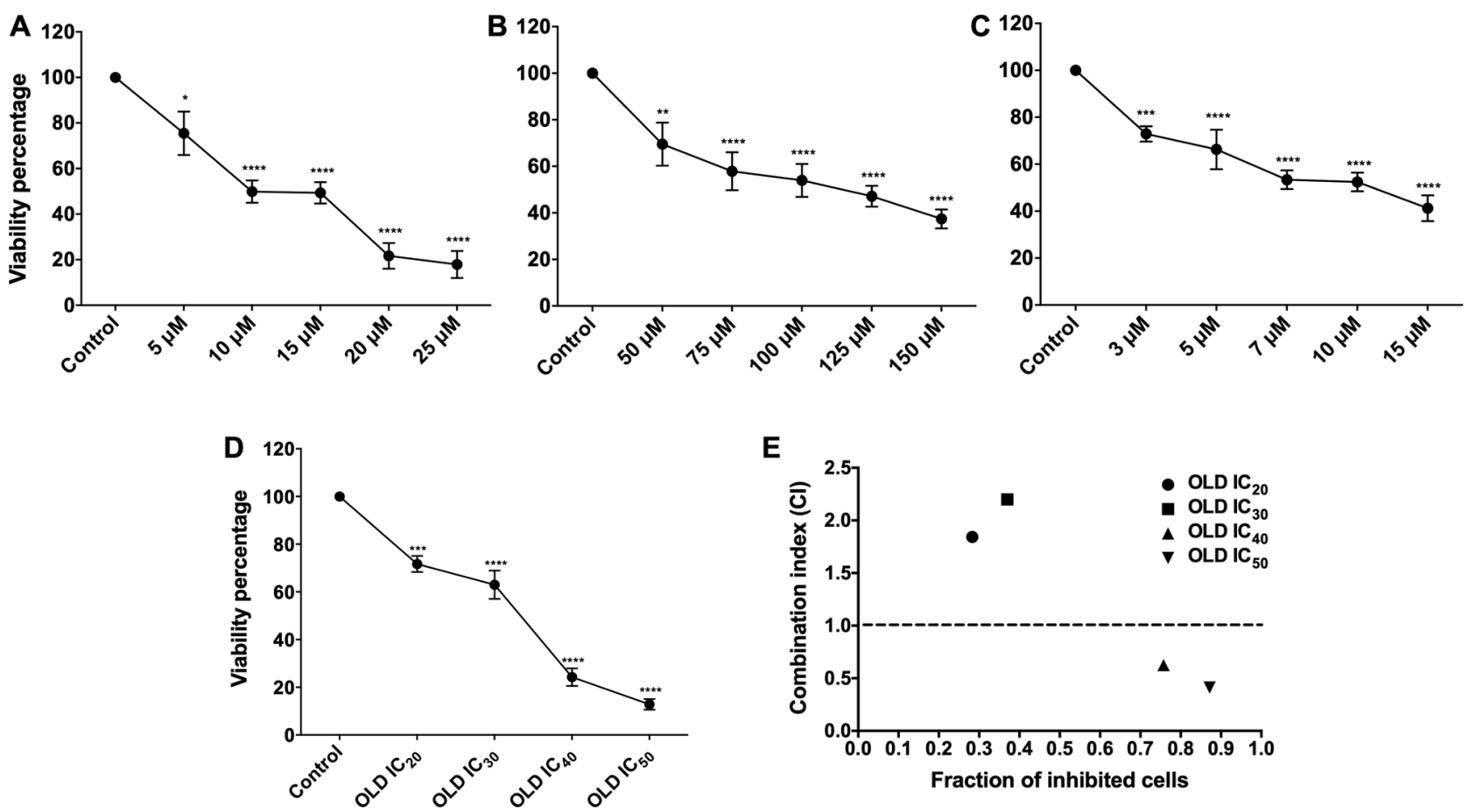

Figure 1. Viability curves of the anti-anabolic compounds and drug synergism evaluation in SW480. Cellular viability was reduced to a greater extent when using low doses of DON alone (C) as compared with either orlistat (A) or lonidamine (B) With the combinations of their $\mathrm{IC}_{20-50}$ doses (D) there was a synergistic effect starting at their $\mathrm{IC}_{40}$ doses $(\mathrm{E}){ }^{*} \mathrm{P}<0.05,{ }^{* * *} \mathrm{P}<0.01,{ }^{* * * *} \mathrm{P}<0.001$ and ${ }^{* * * * *} \mathrm{P}<0.0001$ vs. control. IC, inhibitory concentration; DON, 6-Diazo-5-oxo-L-norleucine; OLD, orlistat + lonidamine + DON .
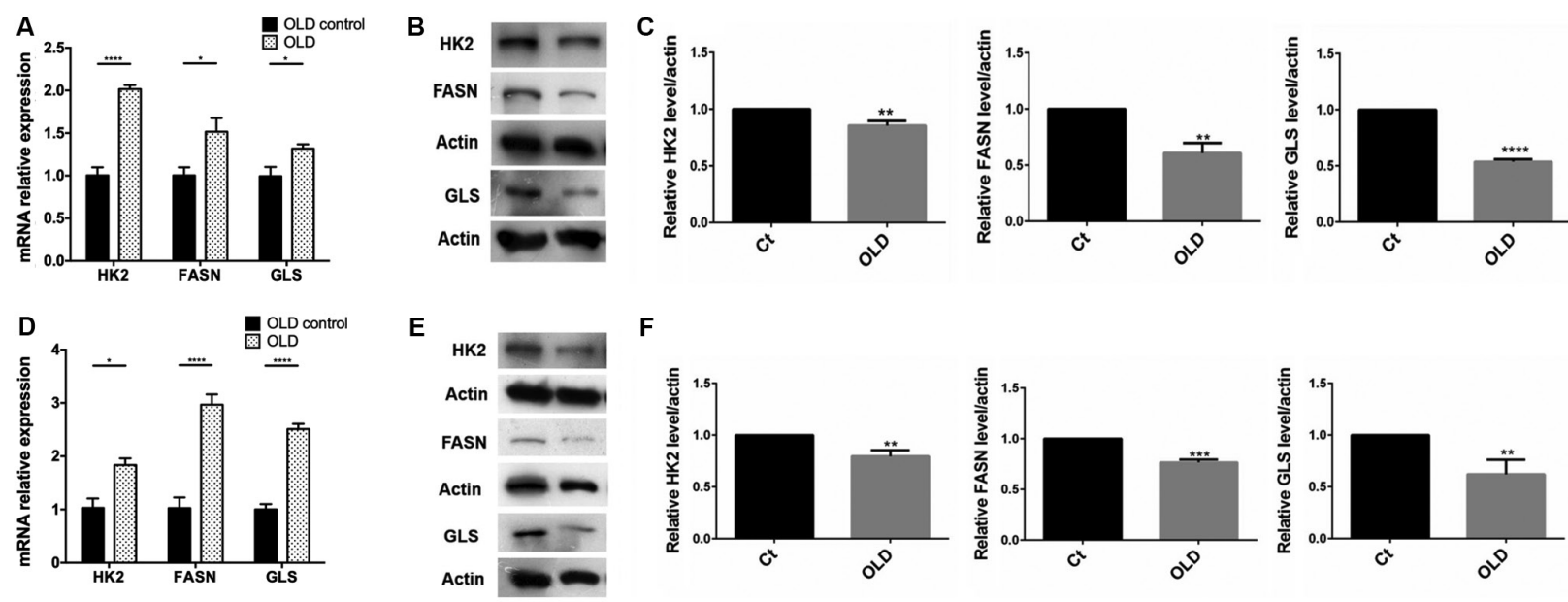

Figure 2. Reverse transcription-quantitative PCR and western blotting of FASN, HK2 and GLS in SW480 and CT26.WT cells treated with OLD. (A) There was a significant increase in mRNA expression of FASN, GLS and particularly HK2 in SW480, and (D) in both FASN and GLS in CT26.WT. (B) Western blot evaluation (C) and densitometry analysis showed a reduction in the intensity of the three targets on SW480, particularly on glutaminase. On the CT26.WT cell line, the reduction in the intensity was more pronounced in FASN (E) western blot evaluation and (F) densitometry analysis. ${ }^{*} \mathrm{P}<0.05,{ }^{* *} \mathrm{P}<0.01,{ }^{* * * *} \mathrm{P}<0.001$ and ${ }^{* * * * *} \mathrm{P}<0.0001$. OLD, orlistat + lonidamine + DON; HK2, hexokinase-II; FASN, fatty acid synthase; GLS, glutaminase; Ct, control.

The pharmacological combination OLD was synergistic at $\mathrm{IC}_{40}$ and $\mathrm{IC}_{50}$. The OLD $\mathrm{IC}_{20}$ and $\mathrm{IC}_{30}$ treatments were antagonistic (Fig. 1E and Table III). However, the $\mathrm{IC}_{40}$ and $\mathrm{IC}_{50}$ treatments were synergistic. The $\mathrm{IC}_{40}$ doses were employed for further assays.

Triple inhibition induces the transcription of the enzyme targets. A significant increase was noted in the relative expression levels of $H K 2, F A S N$ and GLS1 mRNA compared with the control $(\mathrm{P}<0.05)$. The highest expression levels were observed for $H K 2$, followed by $F A S N$ and $G L S 1$ (Fig. 2A) in human cells. The order of overexpression noted in murine cells was as follows: FASN, GLS1 and HK2 (Fig. 2D). Western blot analysis showed a significant decrease of the enzyme targets in human $(\mathrm{P}<0.01$; Fig. $2 \mathrm{~B}$ and $\mathrm{C})$ and murine cells $(\mathrm{P}<0.01$; Fig. 2E and F).

Inhibition of cell viability by downregulation of HK2, FASN and GSL1 gene expression with siRNAs. The comparison of the expression levels of the 3 main target genes in untreated 
Table II. Doses of OLD that achieve $20-50 \%$ reduction in SW480 cell viability.

\begin{tabular}{lccc}
\hline $\begin{array}{l}\text { OLD drug } \\
\text { scheme }\end{array}$ & $\begin{array}{c}\text { Orlistat } \\
\text { dose, } \mu \mathrm{M}\end{array}$ & $\begin{array}{c}\text { Lonidamine } \\
\text { dose, } \mu \mathrm{M}\end{array}$ & $\begin{array}{c}\text { DON } \\
\text { dose, } \mu \mathrm{M}\end{array}$ \\
\hline OLD IC $_{20}$ & 3.59 & 25.1 & 1.92 \\
OLD IC $_{30}$ & 6.01 & 47.36 & 3.62 \\
OLD IC $_{40}$ & 8.7 & 75.86 & 6.12 \\
OLD IC $_{50}$ & 11.7 & 108.4 & 9.95 \\
\hline
\end{tabular}

OLD, orlistat, lonidamine 6-Diazo-5-oxo-L-norleucine; IC, inhibitory concentration.

Table III. Resulting CI values of the pharmacological interactions between $\mathrm{OLD}$ at the $\mathrm{IC}_{20}-\mathrm{IC}_{50}$ combinations.

\begin{tabular}{lcc}
\hline OLD drug scheme & CI value & Pharmacological interaction \\
\hline OLD IC $_{20}$ & 1.843 & Antagonism \\
OLD IC $_{30}$ & 2.2 & Antagonism \\
OLD IC $_{40}$ & 0.625 & Synergism \\
OLD IC $_{50}$ & 0.412 & Synergism \\
\hline
\end{tabular}

OLD, orlistat, lonidamine and 6-Diazo-5-oxo-L-norleucine; IC, inhibitory concentration; CI, combinatory index.

and mock-transfected cells did not exhibit significant differences in both cell lines. A small yet significant reduction in cell viability of $\sim 20 \%$ was noted when each one of the target genes was silenced in both cell lines compared with the effects noted in the scramble transfected cells $(\mathrm{P}<0.01)$. These effects were slightly increased for the FASN gene in SW480 cells (Fig. 3A), whereas depletion of $H K 2$ expression exhibited a significantly higher increase in CT26.WT cells $(\mathrm{P}<0.001$; Fig. 3B). Triple pharmacological inhibition led to a reduction in cell viability of $\sim 80 \%$ (Fig. 1D). Therefore, each one of the siRNA-depleted cells was treated with the two-drug combination that inhibited the two remaining targets. In all the cases, a significant inhibition was noted between siRNA-depleted cells and siRNA-depleted cells with the two-drug combinations $(\mathrm{P}<0.001$ for $H K 2$ and FASN, and $\mathrm{P}<0.05$ for $G L S 1)$ in both cell lines (Fig. 3A and B). These results were verified by western blot analysis (Fig. 3C and D).

Treatment of cells with the OLD combination reduces the expression levels of chemokines and growth factors associated with decreased $p-N F k B-p 65$ protein. The evaluation of the expression levels of the 13 chemokines revealed that 9 of them [interleukin (IL)-9, C-X-C motif chemokine ligand (CXCL) 10, eotaxin, chemokine ligand (CCL) 9, CXCL5, CCL20, CXCL1, CXCL11 and CCL4] exhibited significant reduction in their expression levels $(\mathrm{P}<0.05)$, whereas no change was noted in the remaining 4 (CCL17, CCL2, RANTES and CCL3; Fig. 4A). From all the growth factors investigated, only the expression levels of the stem cell factor (SCF) were decreased (Fig. 4B).
No increase in expression was noted for any of the remaining growth factors. Interestingly, the levels of p-NFkB-p65 showed a significant decrease $(\mathrm{P}<0.01$; Fig. $4 \mathrm{C}$ and $\mathrm{D})$.

\section{Discussion}

The present study shows that the combination of lonidamine, DON and orlistat resulted in a synergistic cytotoxic effect and induced downregulation of their protein targets (HK-2, FASN and GLS). Most likely, as a consequence, the transcription of their target genes was upregulated. Moreover, the genetic or pharmacological depletion of these enzymes reduces cell viability in a cell-dependent manner. It is important to note that 9 out of the 13 chemokines examined have been shown to participate in cancer invasion and metastasis. These molecules were downregulated following treatment, while only the growth factor SCF exhibited reduced expression levels.

A tumor is not only composed of malignant cells. The tumor microenvironment comprises stromal cells, cancer-associated fibroblasts, endothelial cells, pericytes and immune cells (11). The importance of stromal cells in the development of the tumor microenvironment and tumor progression is well known. However, little is known, regarding the ability of the cells from the microenvironment to reprogram their metabolism $(12,13)$.

Previous studies conducted in colon cancer patients analyzed the expression levels of several chemokines in normal and tumor tissues. Erreni et al (14) reported on the mRNA profile of chemokines and chemokine receptors in 8 tumor samples and paired tissues from colon cancer patients and found that the expression of several chemokines was strongly upregulated within the tumor microenvironment; the main identified chemokines were CCL4 and CCL5 and their corresponding receptors CCR1 and CCR5. In another study, the levels of CCL2, CCL4, RANTES, CXCL1, CXCL5 and CXCL8/IL-8 were investigated using ELISA in 10 colorectal carcinomas and their corresponding normal mucosa, and the protein levels of all these chemokines were overexpressed in the tumor counterpart with the exception of RANTES (15).

To the best of our knowledge, this is the first study to demonstrate that a metabolic inhibition with OLD downregulates the expression of several chemokines in colon cancer cells. Among these, IL-9 plays a significant role as an inhibitor of adaptive immunity and prevents the formation of immunologic memory against the growing tumor (16). This highlights the potential for IL-9 neutralization by cancer immunotherapy (16). Zeng et al (17) confirmed that CXCL10 and CXCL11 acted as key protumor chemokines on colon cancer neuroendocrine-like cells. No studies of eotaxin/CCL11 have been conducted in colon cancer, however they were increased in a mouse model of ulcerative colitis, which is considered a colon pre-neoplastic condition (18). Mouse and human colon cancer cells secrete CCL9 and CCL15, respectively and recruit immature myeloid cells, which express the CCL9/15 receptor CCR1 and induce the expression of matrix metalloproteinase (MMP)-2 and MMP-9 enzymes. It is important to note that the CCR1 antagonist BL5923 blocked metastatic colonization and significantly prolonged the survival of tumor-bearing mice (19). The expression levels of CXCL5 were also 
A

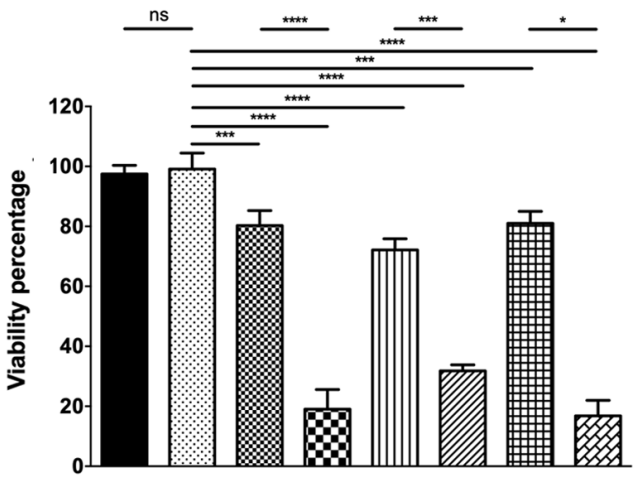

B

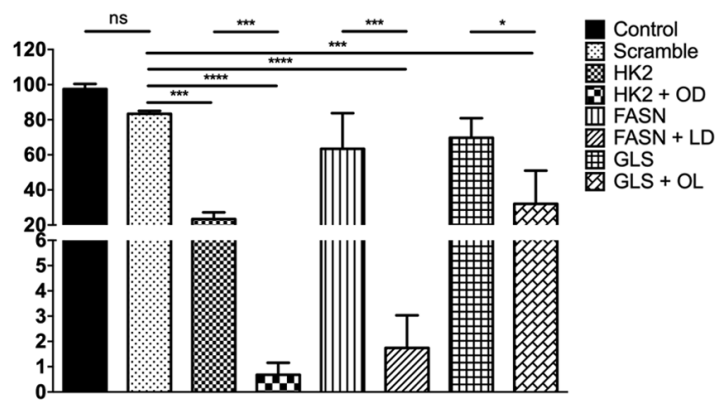

C

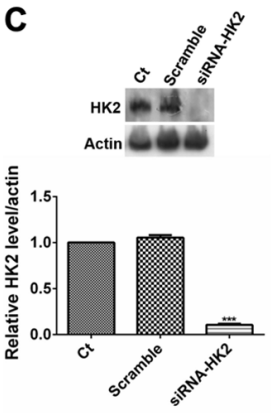

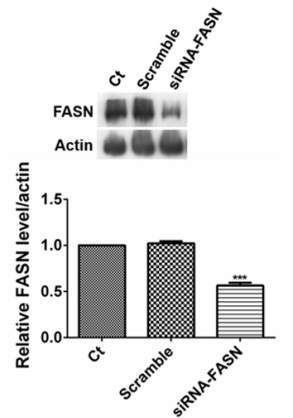
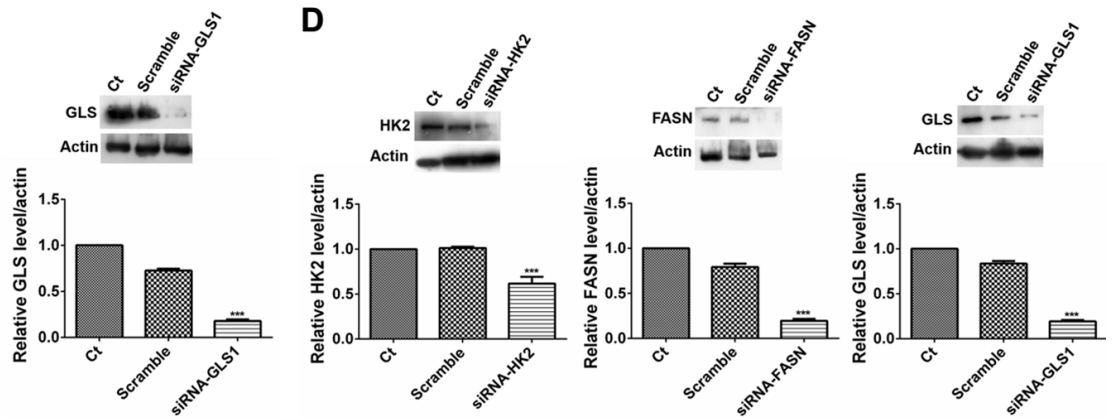

Figure 3. Effect upon cell viability in SW480 and CT26.WT cells undergoing siRNA blockade, with and without anti-metabolic drugs. The downregulation of any of the three targets by siRNAs induced a similar reduction in cell viability in SW480. (A) In CT26.WT, HK2 blockade, either alone or with orlistat + DON, diminished in a more important way the (B) viability. Western blot evaluation showed an important reduction in the intensity of the bands representing the blocked enzymes in (C) SW480 and (D) CT26.WT. ${ }^{*} \mathrm{P}<0.05,{ }^{* * * *} \mathrm{P}<0.001$ and ${ }^{* * * * *} \mathrm{P}<0.0001$. HK2, siRNA against hexokinase-II; HK2 + OD, siRNA against hexokinase-II + orlistat + DON; FASN, siRNA against fatty acid synthase; FASN + LD, siRNA against fatty acid synthase + lonidamine + DON; GLS, siRNA against glutaminase; GLS + OL, siRNA against glutaminase + orlistat + lonidamine; siRNA, small interfering RNA; Ct, control.
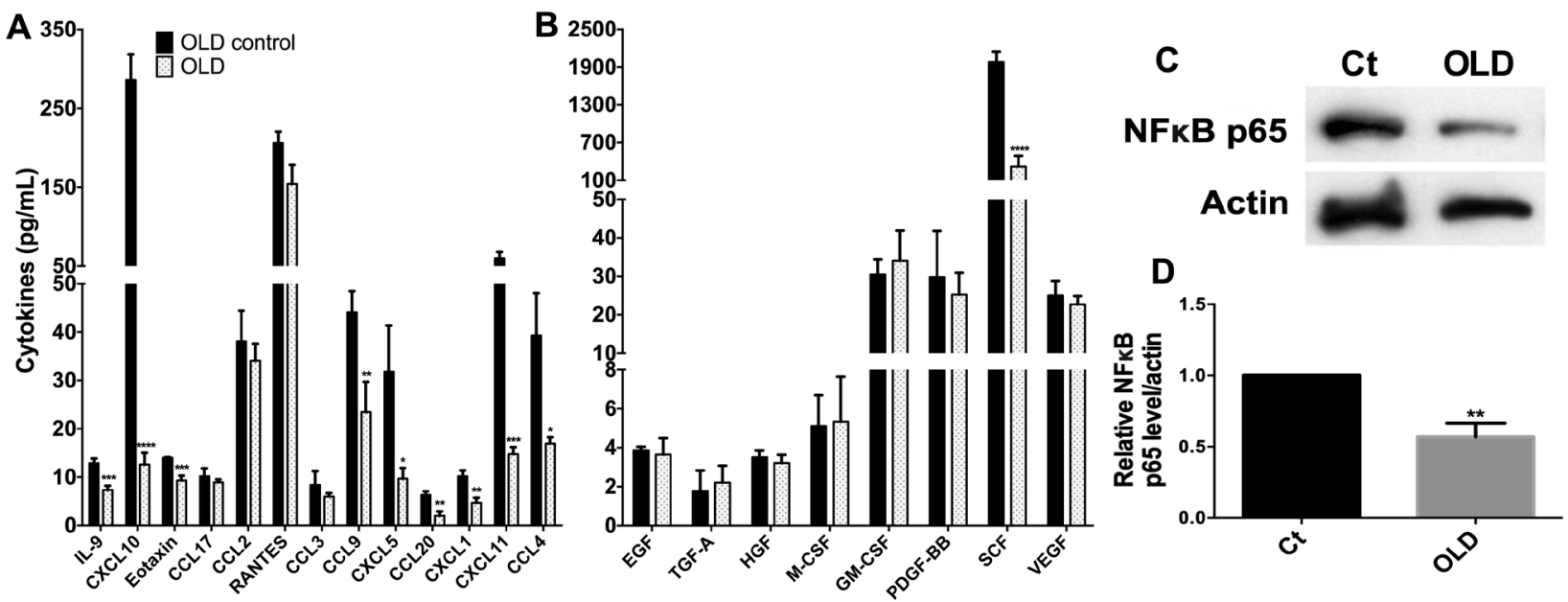

Figure 4. Quantification of chemokines and growth factors in the supernatant of SW480 cells treated with OLD. (A) In the chemokine evaluation, there was an important reduction in IL-9, CXCL10, eotaxin, CCL9, CXCL5, CCL20, CXCL1, CXCL11 and CCL4. (B) Regarding growth factors, only SCF was strongly downregulated. The rest of the cytokines did not differ in a significant way. (C) Western blot evaluation and (D) densitometry analysis showed a reduction in the intensity of the phosphorylated $\mathrm{p} 65$ subunit of NF-kB. ${ }^{*} \mathrm{P}<0.05,{ }^{* *} \mathrm{P}<0.01,{ }^{* * * *} \mathrm{P}<0.001$ and ${ }^{* * * * *} \mathrm{P}<0.0001$. IL, interleukin; OLD, orlistat + lonidamine + DON; Ct, control; NF, nuclear factor; GM-CSF, granulocyte macrophage-colony stimulating factor; TGF, transforming growth factor; SCF, stem cell factor; VEGF, vascular endothelial growth factor; EGF, epidermal growth factor; PDGF-BB, platelet derived growth factor-BB; HGF, hepatocyte growth factor; CCL, chemokine ligand; CXCL, C-X-C motif chemokine ligand.

downregulated in the present study. Cancer cells secrete CXCL5 and in vitro treatment with this ligand can induce protumor effects. Moreover, CXCL5 is elevated in colorectal cancer patients and is considered an independent prognostic factor for adverse effects (20). Yildirim et al (21) demonstrated an increased expression of CXCL5 in colon cancer biopsies, as well as in the serum of these patients compared with the expression levels noted in adenomas and normal epithelia. 
These findings were consistent with the present results. In the present study, the expression levels of CCL20 were downregulated, which has not been previously reported for colon cancer cells or tissues. However, colon cancer patients that exhibit advanced progression of the disease overexpress the receptor CCR6 and treatment with CCL20 increases their migratory and invasive potential (22). In addition, the present study indicated that the expression of CXCL1 was downregulated, which led to an association of the ligand with colon tumor progression. Treatment of SW620 cells with CXCL1 increases their metastatic ability (23). Protumor effects were also reported for CXCL11. This chemokine is overexpressed in colorectal cancer tissues and cell lines, and its downregulation inhibits cell migration and invasion. Moreover, downregulation of CXCL11 has been shown to reduce colorectal cancer cell growth and metastasis in a xenograft model (24). Finally, the present study revealed that CCL4 expression was downregulated by lonidamine, DON and orlistat treatment. CCL4 has been previously shown to participate in tumor progression in concert with CCL2 and CCL3. The expression levels of CCL4 are increased in tumor compared with healthy tissues. The higher levels of CCL4 in plasma are associated with poor prognosis (25). Although the expression levels of CCL2, CCL3, RANTES/CCL5 and CCL17 were not downregulated in the present study, these chemokines can also participate in colon cancer progression (25-29).

The data further demonstrated a decrease in SCF levels. It is known that SCF and its ligand, c-kit, are overexpressed in colon cancer. Both proteins can establish an autocrine c-kit-mediated loop that increases growth, survival, migration and invasive potential (30). This loop further prevents loss of clonogenic potential under differentiation-induced conditions of colon cancer cells (31).

The interpretation of the present results is only hypothesis driven. In the aim to gain a preliminary insight into the potential mechanisms by which OLD treatment reduces chemokines, the phosphorylated p65 subunit of the NFкB pathway was evaluated. $\mathrm{NF} \kappa \mathrm{B}$ is a crucial orchestrator of innate immunity and inflammation as it activates the transcription of target genes, including chemokines among other effectors for inflammation $(32,33)$. Though not demonstrated in this study, the inhibition of NFאB by OLD could be responsible at least indirectly for the reduction of chemokines observed in the present study. More research is needed to demonstrate the mechanisms by which metabolic drugs inhibit activation of $\mathrm{NF} \kappa \mathrm{B}$ which in turn may decrease the expression of chemokines in colon cancer cells.

The present results are preliminary, since the metabolic treatment of cancer cells was analyzed in vitro and the in vivo effect of OLD treatment upon serum chemokines is yet to be evaluated. In conclusion, the results of the present study demonstrated that metabolic antitumor drugs affected the expression levels of chemokines and growth factors associated with a decrease in $\mathrm{NF}-\kappa \mathrm{B}$ activation. Additional studies are required to fully understand the connection between tumor metabolism and chemokine/growth factor regulation.

\section{Acknowledgements}

Not applicable.

\section{Funding}

Alejandro Schcolnik-Cabrera is a PhD student from the Plan de Estudios Combinados en Medicina (UNAM), supported by the CONACyT scholarship (grant no. 439704).

\section{Availability of data and materials}

All data generated or analyzed during the present study are included in this published article.

\section{Authors' contribution}

ASC performed the experiments, analyzed the data and wrote the manuscript. GG performed the western blotting experiments on protein extracts. AB, MY and RB participated in the flow cytometry evaluation of chemokines and growth factors. JD and LC participated in the primer design and in the RT-qPCR experiments. AG conceived the study, reviewed the results and participated in the discussion of the manuscript. All the authors read and approved the final manuscript.

\section{Ethics approval and consent to participate}

The present study was approved by the Ethics Committee of the National Cancer Institute (Mexico City, Mexico). Studies with animal models or with human patients were not performed for this study.

\section{Patient consent for publication}

Not applicable.

\section{Competing interest}

The authors declare absence of conflicts of interest.

\section{References}

1. Pavlova NN and Thompson CB: The emerging hallmarks of cancer metabolism. Cell Metab 23: 27-47, 2016.

2. Kalyanaraman B: Teaching the basics of cancer metabolism: Developing antitumor strategies by exploiting the differences between normal and cancer cell metabolism. Redox Biol 12: 833-842, 2017.

3. Schcolnik-Cabrera A, Chavez-Blanco A, Dominguez-Gomez G and Duenas-Gonzalez A: Understanding tumor anabolism and patient catabolism in cancer-associated cachexia. Am J Cancer Res 7: 1107-1135, 2017.

4. Mathupala SP, Ko YH and Pedersen PL: Hexokinase II: Cancer's double-edged sword acting as both facilitator and gatekeeper of malignancy when bound to mitochondria. Oncogene 25: 4777-4786, 2006.

5. Wise DR and Thompson CB: Glutamine addiction: A new therapeutic target in cancer. Trends Biochem Sci 35: 427-433, 2010.

6. Kridel SJ, Axelrod F, Rozenkrantz N and Smith JW: Orlistat is a novel inhibitor of fatty acid synthase with antitumor activity. Cancer Res 64: 2070-2075, 2004.

7. Cervantes-Madrid D, Dominguez-Gomez G, Gonzalez-Fierro A, Perez-Cardenas E, Taja-Chayeb L, Trejo-Becerril C and Duenas-Gonzalez A: Feasibility and antitumor efficacy in vivo, of simultaneously targeting glycolysis, glutaminolysis and fatty acid synthesis using lonidamine, 6-diazo-5-oxo-L-norleucine and orlistat in colon cancer. Oncol Lett 13: 1905-1910, 2017 ,

8. Cervantes-Madrid D and Dueñas-González A: Antitumor effects of a drug combination targeting glycolysis, glutaminolysis and de novo synthesis of fatty acids. Oncol Rep 34: 1533-1542, 2015. 
9. Matthews H, Deakin J, Rajab M, Idris-Usman $M$ and Nirmalan NJ: Investigating antimalarial drug interactions of emetine dihydrochloride hydrate using CalcuSyn-based interactivity calculations. PLoS One 12: e 0173303, 2017.

10. Livak KJ and Schmittgen TD: Analysis of relative gene expression data using real-time quantitative PCR and the 2(-Delta Delta C(T)) method. Methods 25: 402-408, 2001.

11. Ramamonjisoa $\mathrm{N}$ and Ackerstaff $\mathrm{E}$ : Characterization of the tumor microenvironment and tumor-stroma interaction by non-invasive preclinical imaging. Front Oncol 7: 3, 2017.

12. Tuccitto A, Shahaj E, Vergani E, Ferro S, Huber V, Rodolfo M, Castelli C, Rivoltini L and Vallacchi V: Immunosuppressive circuits in tumor microenvironment and their influence on cancer treatment efficacy. Virchows Arch 474: 407-420, 2018.

13. Wegiel B, Vuerich M, Daneshmandi S and Seth P: Metabolic switch in the tumor microenvironment determines immune responses to anti-cancer therapy. Front Oncol 8: 284, 2018.

14. Erreni M, Bianchi P, Laghi L, Mirolo M, Fabbri M, Locati M, Mantovani A and Allavena P: Expression of chemokines and chemokine receptors in human colon cancer. Methods Enzymol 460: 105-121, 2009.

15. Baier PK, Eggstein S, Wolff-Vorbeck G, Baumgartner U and Hopt UT: Chemokines in human colorectal carcinoma. Anticancer Res 25: 3581-3584, 2005.

16. Hoelzinger DB, Dominguez AL, Cohen PA and Gendler SJ: Inhibition of adaptive immunity by IL9 can be disrupted to achieve rapid T-cell sensitization and rejection of progressive tumor challenges. Cancer Res 74: 6845-6855, 2014.

17. Zeng YJ, Lai W, Wu H, Liu L, Xu HY, Wang J and Chu ZH: Neuroendocrine-like cells -derived CXCL10 and CXCL11 induce the infiltration of tumor-associated macrophage leading to the poor prognosis of colorectal cancer. Oncotarget 7: 27394-27407, 2016.

18. Coburn LA, Horst SN, Chaturvedi R, Brown CT, Allaman MM, Scull BP, Singh K, Piazuelo MB, Chitnavis MV, Hodges ME, et al: High-throughput multi-analyte Luminex profiling implicates eotaxin-1 in ulcerative colitis. PLoS One 8: e82300, 2013.

19. Kitamura T, Fujishita T, Loetscher P, Revesz L, Hashida H, Kizaka-Kondoh S, Aoki $\mathrm{M}$ and Taketo MM: Inactivation of chemokine (C-C motif) receptor 1 (CCR1) suppresses colon cancer liver metastasis by blocking accumulation of immature myeloid cells in a mouse model. Proc Natl Acad Sci USA 107: 13063-13068, 2010.

20. Kawamura M, Toiyama Y, Tanaka K, Saigusa S, Okugawa Y, Hiro J, Uchida K, Mohri Y, Inoue Y and Kusunoki M: CXCL5, a promoter of cell proliferation, migration and invasion, is a novel serum prognostic marker in patients with colorectal cancer. Eur J Cancer 48: 2244-2251, 2012.

21. Yildirim K, Colak E, Aktimur R, Gun S, Taskin MH, Nigdelioglu A, Aktimur SH, Karagöz F and Ozlem N: Clinica value of CXCL5 for determining of colorectal cancer. Asian Pac J Cancer Prev 19: 2481-2484, 2018.
22. Kapur N, Mir H, Clark Iii CE, Krishnamurti U, Beech DJ, Lillard JW and Singh S: CCR6 expression in colon cancer is associated with advanced disease and supports epithelial-to-mesenchymal transition. Br J Cancer 114: 1343-1351, 2016.

23. Hsu YL, Chen YJ, Chang WA, Jian SF, Fan HL, Wang JY and Kuo PL: Interaction between tumor-associated dendritic cells and colon cancer cells contributes to tumor progression via CXCL1. Int J Mol Sci 19: E2427, 2018.

24. Gao YJ, Liu L, Li S, Yuan GF, Li L, Zhu HY and Cao GY: Down-regulation of CXCL11 inhibits colorectal cancer cell growth and epithelial-mesenchymal transition. Onco Targets Ther 11: 7333-7343, 2018.

25. De la Fuente Lopez M, Landskron G, Parada D, Dubois-Camacho K, Simian D, Martinez M, Romero D, Roa JC, Chahuán I, Gutiérrez R, et al: The relationship between chemokines CCL2, CCL3, and CCL4 with the tumor microenvironment and tumor-associated macrophage markers in colorectal cancer. Tumour Biol 40: 1010428318810059, 2018.

26. Al-haidari AA, Syk I, Jirstrom K and Thorlacius H: CCR4 mediates CCL17 (TARC)-induced migration of human colon cancer cells via RhoA/Rho-kinase signaling. Int J Colorectal Dis 28: 1479-1487, 2013.

27. Cambien B, Richard-Fiardo P, Karimdjee BF, Martini V,Ferrua B, Pitard B, Schmid-Antomarchi H and Schmid-Alliana A: CCL5 neutralization restricts cancer growth and potentiates the targeting of PDGFR $\beta$ in colorectal carcinoma. PLoS One 6: e28842, 2011.

28. Roblek M, Strutzmann E, Zankl C, Adage T, Heikenwalder M, Atlic A, Weis R, Kungl A and Borsig L: Targeting of CCL2-CCR2-glycosaminoglycan axis using a CCL2 decoy protein attenuates metastasis through inhibition of tumor cell seeding. Neoplasia 18: 49-59, 2016.

29. Tanabe Y, Sasaki S, Mukaida N and Baba T: Blockade of the chemokine receptor, CCR5, reduces the growth of orthotopically injected colon cancer cells via limiting cancer-associated fibroblast accumulation. Oncotarget 7: 48335-48345, 2016.

30. Bellone G, Carbone A, Sibona N, Bosco O, Tibaudi D, Smirne C, Martone T, Gramigni C, Camandona M, Emanuelli G and Rodeck U: Aberrant activation of c-kit protects colon carcinoma cells against apoptosis and enhances their invasive potential. Cancer Res 61: 2200-2206, 2001.

31. Fatrai S, van Schelven SJ, Ubink I, Govaert KM, Raats D, Koster J, Verheem A, Borel Rinkes IH and Kranenburg O: Maintenance of clonogenic KIT(+) human colon tumor cells requires secretion of stem cell factor by differentiated tumor cells. Gastroenterology 149: 692-704, 2015.

32. Ghosh S and Karin M: Missing pieces in the NF-kappaB puzzle. Cell 109 (Suppl): S81-S96, 2002.

33. Del Prete A, Allavena P, Santoro G, Fumarulo R, Corsi MM and Mantovani A: Molecular pathways in cancer-related inflammation. Biochem Med (Zagreb) 21: 264-275, 2011. 